(high score indicating wound complications, maximum 41). Mean satisfaction score amongst parents was 4.3 (out of 5). Conclusion RWC's are desirable amongst our patient cohort. Parents engaged well with the remote service and satisfaction was high. Following this pilot we propose expansion of this service to empower families in the COVID-era and beyond.

\section{RESEARCHING SARS-COV-2 FROM HOME: MAXIMISING THE BENEFIT OF THE GOSH DIGITAL RESEARCH ENVIRONMENT DURING NATIONAL LOCKDOWN}

${ }^{1}$ Richard Issitt, ${ }^{1}$ John Booth, ${ }^{1}$ Lydia Briggs, ${ }^{1}$ William Bryant, ${ }^{1}$ Mohsin Shah, ${ }^{1}$ Anastassia Spiridou, ${ }^{2}$ Neil J Sebire. ${ }^{1} D R I V E$, Great Ormond Street Hospital for Children NHS Foundation Trust; ${ }^{2}$ GOSH DRIVE

\subsection{6/archdischild-2020-gosh. 10}

The 2019 novel severe acute respiratory syndrome coronavirus 2 (SARS-CoV-2) causes COVID-19, characterised by potentially severe respiratory and gastrointestinal symptoms. As of early October 2020, there were around 34 million confirmed cases of COVID-19 and more than 1,000,000 associated deaths globally. Initial data indicated that COVID-19 affected children with fewer symptoms and less severe disease compared with adults, however more recently some have developed an unusual associated systemic inflammatory condition: paediatric inflammatory multisystem syndrome temporally related to SARS-CoV-2 infection (PIMS-TS).

In the UK, Public Health England outlined a shielding strategy designed to protect those extremely vulnerable to SARS-CoV-2 infection, such as individuals who are immunocompromised. At the same time, the UK Government enforced a National Lockdown for all except key workers. As such, researchers were unable to continue working on site at hospitals and Universities. The web-based Digital Research Environment platform at GOSH made it possible for research on GOSH patients to continue despite those limitations. Even more, the efficient data integration processes that the DRE have implemented enabled a rapid extraction of COVID-19 related data from the GOSH EPR system to support initial research results.

We were able to examine a cohort of paediatric patients, presenting to GOSH, with suspected COVID-19 or PIMS-TS to document their clinical characteristics and outcomes with regard to the presence of underlying medical conditions associated with vulnerability and to establish whether underlying conditions such as vitamin D deficiency were prominent in these patients. By analysing this data during the first wave, we were able to identify that apparently 'vulnerable' groups were not overrepresented in patients hospitalised with COVID-19 or PIMS-TS.

\section{TOWARDS INTERNATIONAL FEDERATED HEALTH DATA ANALYSIS: EXPERIENCE WITH THE 4CE CONSORTIUM FOR CHARACTERISATION OF COVID-19}

${ }^{1}$ Anastassia Spiridou, 'John Booth, ${ }^{1}$ Richard Issitt, ${ }^{1}$ Mohsin Shah, ${ }^{1}$ William Bryant, ${ }^{2} \mathrm{Nei}$ J Sebire. ${ }^{1}$ DRIVE, Great Ormond Street Hospital for Children NHS Foundation Trust; ${ }^{2}$ GOSH DRIVE

10.1136/archdischild-2020-gosh.11
The Covid-19 pandemic necessitated collaborations from hospitals around the world to improve knowledge and find suitable treatments. Responding to the quick spread of the virus, an international consortium was formed (4CE, https://covidclinical.net/) to rapidly collect data from the Electronic Patient Records (EPRs) of participating hospitals and publish results. The Digital Research Environment (DRE) enabled GOSH to join the consortium as one of the lead contributors of paediatric Covid-19 data in Europe and the only hospital in the U.K. The objective was to develop methods that ensure quick data collection and analysis using a mixed model of federated and centralised data analysis, and publish results on the clinical characterisation of Covid-19 in both children and adults to inform care and research.

Building on the DRE's automated data integration processes and flows from the GOSH EPR system, we developed methods to semi-automate our Covid-19 cohort identification, restructure and harmonise our data to the internationally agreed specification and standards, perform quality control collaboratively, produce aggregate-level data using summary statistics, validate severity, and incorporate appropriate obfuscation of results. GOSH IG approval was obtained before sharing summary data with 4CE for further analysis.

We were able to rapidly develop suitable technical and governance solutions as a first step towards federated health data analysis in an international collaborative of hospitals. The consortium analysis results, currently being submitted for publication, clinically characterise Covid-19 at an international level informing care and research. 4CE offers a new model of collaborative health data analysis based on the use of EPRs and federated analysis to respond quickly to pandemics like Covid-19.

The use of GOSH DRE enabled our participation in the 4CE consortium and quick generation of collaborative data analysis results on Covid-19 paving the way for further development of advanced international federated health data analytics using EPRs.

\section{COVID-19; A REFLECTION OF PHYSIOTHERAPY INTERVENTION IN THE PICU}

Helen Mercer. Great Ormond Street Hospital

\subsection{6/archdischild-2020-gosh.12}

The novel COVID-19 poses challenges in the paediatric physiotherapy community. In March 2020 there were not, and still are not, any clinical guidelines on the management of the physiotherapy needs in this cohort of children.

Links between national adult and paediatric services were formed to utilise and adapt adult national intensive care guidelines to plan paediatric COVID-19 respiratory physiotherapy assessment and treatments. With support of medical colleagues, protocols on the use of humidifiers, ventilators and bagging circuit filters and the necessity of closed suction were created.

To determine a suggested management, we utilised knowledge of viral/ARDS conditions and devised an algorithm to identify which patients required physiotherapy intervention.

Proning of patients was identified as a key treatment. Together with our clinical simulation centre, a skills lab was 